\title{
ISING MODEL ON TANGLED CHAIN. II. MAGNETIZATION AND SUSCEPTIBILITY
}

\author{
R. MEJDANI \\ International Centre for Thcorctical Physics, Trieste 34100, Italy \\ and \\ Department of Physics, University of Tirana, Tirana, Albania*
}

(Received February 11, 1994)

\begin{abstract}
In the preceding paper we have considered an Ising model defined on tangled chain to study the behaviour of the frce energy and entropy, particularly in the zero-field and zero-temperature limit. In this paper, following the main line and basing on some results of the previous work, we shall study in the "language" of state configurations the behaviour of the magnetization and the susceptibility for different conditions of the model, to understand better the competition between the ferromagnetic bonds along the chain and the antiferromagnetic additional bonds across the chain. Particularly interesting is the beliaviour of the susceptibility in the zero-field and zero-temperature limit. Exact solutions for the magnetization and susceptibility, generated by analytical calculations and iterative algorithms, are described. The additional bonds, introduced as a form of perfect disorder, indicate a particular effect on the spin correlation. We found that the condition $J=-J^{\prime}$ between the ferromagnetic interaction $J$ along the chain and the antiferromagnetic interaction $J^{\prime}$ across the chain is somewhat as a "transition-region" condition for this behaviour.
\end{abstract}

PACS numbers: $65.50 .+\mathrm{m}, 75.10 .11 \mathrm{k}, 75.10 . \mathrm{Nr}, 05.50 .+\mathrm{q}$

\section{Introduction}

In the preceding paper [1], which we refer to as I hereafter, we have studied for the Ising model defined on tangled chain the behaviour of the free energy and entropy, using a modified transfer matrix method [1-3], some simple analytical calculations and iterative algorithms [1,3]. IIere we are concerned with the behaviour of the magnetization and susceptibility, basing on our previous results in I about the free energy, internal energy in zero-ficld and zero-temperature limit, entropy and their "transition region". Studying the behaviour of the susceptibility in the zero-field and zero-temperature limit, we will see that the additional bonds, introduced as a form of perfect disorder, can affect the spin correlation breaking up the

* Permanent address. 
magnetic chain into independent segments, as in the case of an Ising-like magnetic chain diluted with nonmagnetic impurities [4]. The tentative for an interpretation of this behaviour in terms of uneven effective local temperature $[2,5]$ represents for us some problems, particularly beyond the "transition region". We shall try to give its interpretation through the analysis of ground-state configurations and the ground-state degeneracies [6-12].

\section{Description of the model}

As in I, consider ferromagnetic Ising spins placed on a tangled chain with a coupling constant $-J(J>0)$ between the nearest neighbours along the chain and with a coupling constant $-J^{\prime}\left(J^{\prime}>0, J^{\prime}=0\right.$ or $\left.J^{\prime}<0\right)$ across the chain. We suppose that there are $n_{\mathrm{d}}$ additional bonds or "long range" bonds $J^{\prime}$, uniformly distributed along the chain. Between the two opposite ends of each long range bond, there are $n_{1}$ spins. There are $n_{2}$ spins between one end of a long range bond and the beginning of the next long range bond. These parameters of chain configuration are then related to the total number of spins by $N=n_{\mathrm{d}}\left(n_{1}+n_{2}+2\right)$, where the number 2 is to count those spins coupled by the long range bonds.

The Hamiltonian of the system is

$$
H(S)=H o(S)+H^{\prime}(S)
$$

where

$$
H \circ(S)=-J \sum_{\mathrm{nn}} S_{i} S_{j}-B \sum_{i} S_{i}, \quad I^{\prime}(S)=-J^{\prime} \sum_{\mathrm{dn}} S_{i} S_{j} .
$$

$B$ is the magnetic field and $S_{i}= \pm 1$. The pure system Uamiltonian $H o(S)$ represents the coupling of all spins with their nearest neighbours (nn) along the chain and the "perturbation" term $I I^{\prime}(S)$ considers additional bonds between selected pairs of distant neighbours (dn). While the first term in $I I$ favours ferromagnetic alignment of the spins $(J>0)$, the second favours alignment with the sign of the additional bonds. The competition between these terms is the source of much of the complexity of the model.

As we know from I, for a segment of $(n+2)$ spins, the traced middle part over $S_{2} \ldots S_{n+1}$ leaves for two spins $S_{1}, S_{n+2}$ on the opposite ends of the segment, a temperature dependent constant $c[n]$, an effective field $y[n]$ and an effective coupling $x[n]$, generated from $c[0], y[0], x[0]$ (i.e., from a two-spin segment which has no middle part) by the recurrence relations

$$
\begin{aligned}
& c[n]=2(y[0] y[n-1]+c[0] c[n-1]), \\
& y[n]=2(y[0] x[n-1]+c[0] y[n-1]), \\
& x[n]=2(x[0] x[n-1]+y[0] y[n-1]),
\end{aligned}
$$

with

$$
\begin{aligned}
& c[0]=0.5\left[k \operatorname{ch}(f / t)+k^{-1}\right], \\
& y[0]=0.5 k \operatorname{sh}(f / t), \\
& x[0]=0.5\left[k \operatorname{ch}(f / t)-k^{-1}\right],
\end{aligned}
$$


where $t=\left(k_{\mathrm{B}} T / J\right)\left(k_{\mathrm{B}}-\right.$ the Boltzmann constant and $T-$ the absolute temperature) and $f=(B / J)$ are the reduced temperature and the reduced field, respectively, and $k=\exp (1 / t)$. Using the same symbols as in I, the elements of the transfer matrix $T$ for a "box", composed of a tangled part of $n_{1}+2$ spins and an untangled part of $n_{2}$ spins are

$$
\begin{aligned}
& T_{++} \equiv A=a[n 1] a[n 2]+b[n 1] b[n 2]=k^{\prime} a_{e} a_{0}+k^{\prime-1} b_{e} b_{0}, \\
& T_{+-} \equiv B=a[n 1] b[n 2]+b[n 1] d[n 2]=k^{\prime} a_{e} b_{0}+k^{\prime-1} b_{e} d_{0}, \\
& T_{-+} \equiv C=b[n 1] a[n 2]+d[n 1] b[n 2]=k^{\prime-1} b_{e} a_{0}+k^{\prime-1} d_{e} b_{0}, \\
& T_{--} \equiv D=b[n 1] b[n 2]+d[n 1] d[n 2]=k^{\prime-1} b_{e} b_{0}+k^{\prime} d_{e} d_{0},
\end{aligned}
$$

where $k^{\prime}=\exp \left(J^{\prime} / k_{\mathrm{B}} T\right)=\exp (e / t)$ with $e=J^{\prime} / J$, and

$$
\begin{aligned}
& a_{q}=c[j]+2 y[j]+x[j], \\
& b_{q}=c[j]-x[j], \\
& d_{q}=c[j]-2 y[j]+x[j],
\end{aligned}
$$

with the symbol combinations: $\left(q=e, j=n_{1}\right)$ and $\left(q=0, j=n_{2}\right)$.

For the larger eigenvalue of the transfer matrix we have

$$
\lambda_{\max } \equiv \lambda_{+}=0.5\left[(A+D)+\sqrt{(A-D)^{2}+4 B C}\right] \text {. }
$$

Since the maximum eigenvalue $\lambda_{\max }$ is equal to the grand partition function per box (or $n_{1}+n_{2}+2$ sites), in the thermodynamic linit, the thermodynamic potential or the free encrgy per site is derived as

$$
\frac{F_{N}}{N}=-k_{\mathrm{B}} T \lim _{N \rightarrow \infty}\left[N^{-1} \ln \left(\lambda_{\max }\right)^{N /(n 1+n 2+2)}\right]=-\frac{k_{\mathrm{I}} T}{n_{1}+n_{2}+2} \ln \lambda_{\max } .
$$

\section{The magnetization}

\subsection{The simple case $\left(n_{1}=n_{2}=1\right)$}

From (3)-(4) we obtain

$$
\begin{aligned}
& c[1]=0.5\left[k^{2} \operatorname{ch}(2 f / l)+2 \operatorname{ch}(f / l)+k^{-2}\right], \\
& y[1]=0.5\left[k^{2} \operatorname{sh}(2 f / t)\right], \\
& x[1]=0.5\left[k^{2} \operatorname{ch}(2 f / l)-2 \operatorname{ch}(f / l)+k^{-2}\right],
\end{aligned}
$$

while from (6) and (9) we have

$$
\begin{aligned}
& a_{e}=a_{0}=k^{2} \exp (2 f / t)+k^{-2}, \\
& b_{e}=b_{0}=2 \operatorname{ch}(f / t), \\
& d_{e}=a_{0}=k^{2} \exp (-2 f / t)+k^{-2} .
\end{aligned}
$$


Substituting these expressions to (5), the elements of the transfer matrix in this simple case will be

$$
\begin{aligned}
& A=k^{\prime}\left[k^{2} \exp (2 f / l)+k^{-2}\right]^{2}+k^{\prime-1}[2 \operatorname{ch}(f / l)]^{2}, \\
& B=2 \operatorname{ch}(f / l)\left\{k^{\prime}\left[k^{2} \exp (2 f / l)+k^{-2}\right]+k^{\prime-1}\left[k^{2} \exp (-2 f / t)+k^{-2}\right]\right\}, \\
& C=2 \operatorname{ch}(f / t)\left\{k^{\prime-1}\left[k^{2} \exp \left(2 \int / l\right)+k^{-2}\right]+k^{\prime}\left[k^{2} \exp (-2 f / t)+k^{-2}\right]\right\}, \\
& D=k^{\prime-1}[2 \operatorname{ch}(f / l)]^{2}+k^{\prime}\left[k^{2} \exp \left(-2 \int / l\right)+k^{-2}\right]^{2} .
\end{aligned}
$$

From (8) the magnelization per spin is

$$
m=\left[t /\left(n_{1}+n_{2}+2\right)\right]\left(\lambda_{\max }^{\prime} / \lambda_{\max }\right),
$$

where $\lambda_{\max }^{\prime}=\partial \lambda_{\max } / \partial f$ represents now the first derivative of $\lambda_{\max }$ related to the reduced field $f$. Denoting with' this type of derivative (in I the ' was used for the first derivatives with respect to the reduced temperature $t$ ), for the case $n_{1}=n_{2}=1$, we obtain

$$
\begin{aligned}
A^{\prime}= & \left.(4 / t)\left[k^{\prime} k^{4} \exp (4 f / l)+k^{\prime} \exp (2 f / t)+k^{\prime-1} \operatorname{sh}(2 f / t)\right]\right] \\
B^{\prime}= & (2 / t)\left\{\left[k^{\prime}\left(k^{2} \exp (2 f / l)+k^{-2}\right)+k^{\prime-1}\left(k^{2} \operatorname{cxp}(-2 f / \iota)+k^{-2}\right)\right] \operatorname{sh}(f / l)\right. \\
& \left.+\left[k^{\prime} k^{2} \exp (2 f / t)-k^{\prime-1} k^{2} \exp (-2 f / \iota)\right] 2 \operatorname{ch}(f / t)\right\} \\
C^{\prime}= & (2 / t)\left\{\left[k^{\prime-1}\left(k^{2} \exp (2 f / t)+k^{-2}\right)+k^{\prime}\left(k^{2} \exp (-2 f / t)+k^{-2}\right)\right] \operatorname{sh}(f / t)\right. \\
& \left.+\left[k^{\prime-1} k^{2} \exp (2 f / t)-k^{\prime} k^{2} \exp (-2 f / l)\right] 2 \operatorname{ch}(f / t)\right\}, \\
D^{\prime}= & (4 / t)\left[k^{\prime-1} \operatorname{sh}(2 f / \iota)-k^{\prime} k^{4} \exp (-4 f / l)-k^{\prime} \exp (-2 f / t)\right] .
\end{aligned}
$$

Considering (11)-(13) and the expression

$$
\begin{aligned}
\lambda_{\max }^{\prime}= & 0.5\left\{\left(A^{\prime}+D^{\prime}\right)\right. \\
& \left.+\left[(A-D)\left(A^{\prime}-D^{\prime}\right)+2\left(B^{\prime} C^{\prime}+B C^{\prime}\right)\right] / \sqrt{(A-D)^{2}+4 B C}\right\},
\end{aligned}
$$

we can study the behaviour of the magnetization $m$, for different types of additional interactions (ferromagnetic and antiferromagnetic) and different values of the magnetic field. In the particular case $n_{1}=n_{2}=1$ and $e=0$ we reobtain for $m$ the usual (unperturbed chain) result [13]: $m=\operatorname{sh}(f / t) / \sqrt{\operatorname{sh}^{2}(f / t)+\exp (-4 / t)}$, while in the zero-temperature limit $m=0$ at $f=0$.

In Figs. 1-4 we have represented some of $(m-t)$ diagrams for different values of $e$ and $f$. To give the right interpretation of these diagrams we will reconsider results of paper I for the free cnergy and the entropy per spin in the zero-field and zero-temperature limit. Therefore for $t \rightarrow 0$ we had

$$
\begin{aligned}
& F \approx-[(4+e) / 4], \quad S \rightarrow 0(e>-1), \\
& F \approx-3 / 4, \quad S \rightarrow[(\ln 3) / 4](e=-1), \\
& F \approx-[(2-e) / 4], \quad S \rightarrow[(\ln 2) / 4](e<-1) .
\end{aligned}
$$




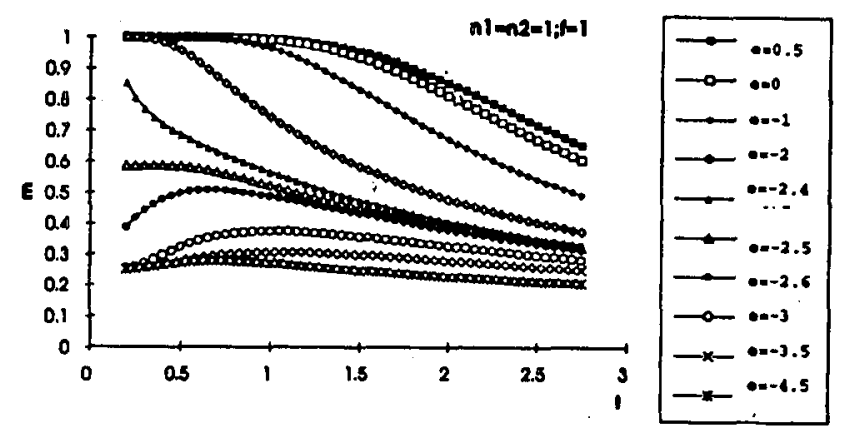

Fig. 1. Magnetization $m$ as a function of reduced temperalure $t$ in the case $n_{1}=n_{2}=1$ and $f=1$, for different values of $e(c=0.5,0,-1,-2,-2.4,-2.5,-2.6,-3,-3.5,-4.5)$.

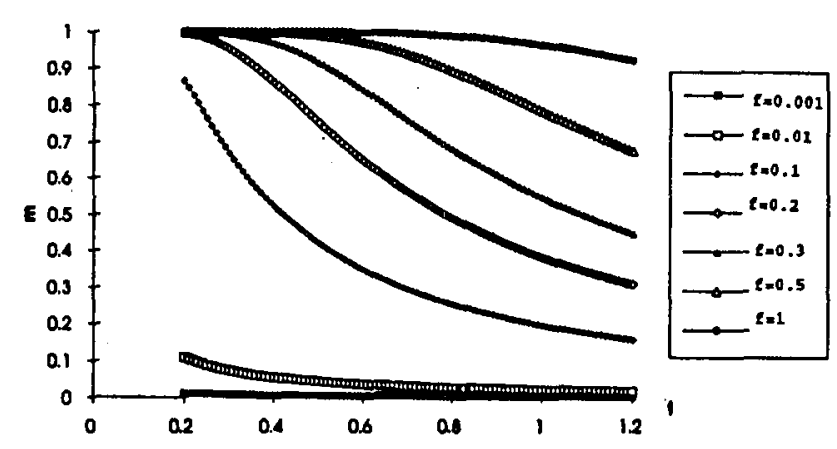

Fig. 2. $m$ as a function of $t$ in the case $n_{1}=n_{2}=1$ for a particular value of $e(e=-1)$ and different values of reduced filed $f(f=0.001,0.01,0.1,0.2,0.3,0.5,1)$.

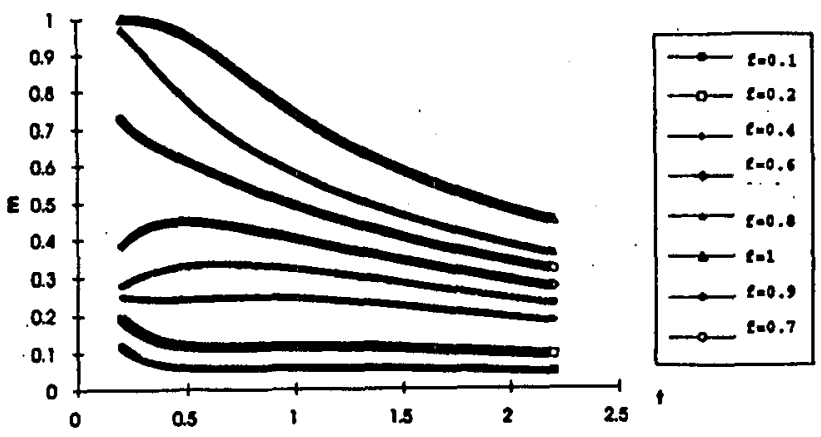

Fig. 3. (m-t) diagrams for the configuration $n_{1}=1, n_{2}=1(e=-2$ and $f=$ $0.1,0.2,0.4,0.6,0.7,0.8,0.9,1)$. 


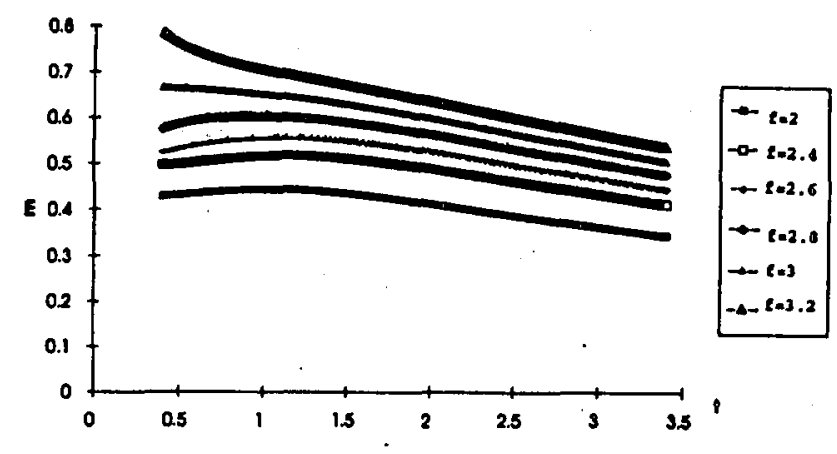

Fig. 4. (m-l) diagrams for the configurations $n_{1}=n_{2}=1$ and "extreme" values of $e$ $(c=-5)$ and $f(f=2,2.4,2.6,2.8,3,3.2)$.

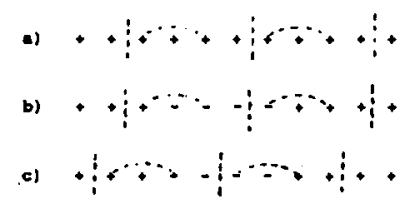

Fig. 5. Schematic presentation of the ground-state configurations in the zero field and zero temperature. The + represents the "up" spin $(S=+1)$ and the - the "down" spin $(S=-1)$.

From these results we can represent schematically the ground state configurations in three different cases $(e>-1, e=-1, e<-1)$ (Fig. 5). For $e>-1$ the only possible configuration is a (all spins align in the same direction, so that there is a unique "metastable" state, with all spins pointing in the same direction, either +1 or -1 ). For $e=-1$ there are three degenerate configurations $a$, $b, c$, all of which have the same energy (hence the ground-state entropy for a "box" of 4 spins is $\ln 3$ ). The configurations $b$ and $c$ have also unstable domain boundaries. For $e<-1$ the possible configurations are $b$ and $c$ (in this case the degeneracy is two).

Following the same way as in I, considering the "highest powcr" terms in the expression of $F$ in the zero-temperature limit (with $f \neq 0$ ) for the free energy per spin $F$ at $l=0$ (or for the internal energy per spin) we have found

$$
\begin{aligned}
& F=-[(4+e+4 f) / 4](e>-1, f>0), \\
& F=-[(3+4 f) / 4](e=-1, f>0), \\
& F=-[(4+e+4 f) / 4],-[(-e+2 f) / 4], \\
& \quad-[(2+3 f) / 4],-[(2-e+f) / 4](e<-1, f>0) .
\end{aligned}
$$

In the last case the real configuration will be the configuration with the minimum value of energy (which corresponds to the "highest powcr" term) for $e<-1$ 
and different value of $f(f>0)$. In some particular cases the real configuration could be a combination of ground states with the same energy (but different magnetization). We may note that the ground state configurations are very sensitive to changes in the magnetic field and each metastable state is characterized by its energy per spin and its magnetization per spin (reflected in terms of $f$ ).

Considering these configurations we can explain properly the different $(m-t)$ diagrams. Therefore in Fig. 1 the five upper curves (from $e=0.5$ to $e=-2.4$ ) for $f=1$ correspond at $t=0$ to the configuration with energy per spin $-[(4+e+f) / 4]$, which means that the magnetization $m$ per spin at $t=0$, in the presence of the magnetic ficld is $m=1$. The four lower curves (from $e=-2.6$ to $e=-4.5)$ correspond to the configuration with energy per spin $-[(2-e+f) / 4]$, which means that the zero-temperature magnetization per spin and per box (per $n_{1}+n_{2}+2=4$ spins) in these cases is 0.25 and 1 , respectively. Also, the magnetization passes through a maximum for $t \neq 0$. The curve for $e=-2.5$ corresponds to a combination of two configurations with energies $-[(4+e+4 f) / 4]$ and $-[(2-e+f) / 4]$, and the zero-temperature magnetization per spin is between 1 and $0.25(m \approx 0.6)$. In the same way one can interpret the other diagrams in Figs. 2, 3, 4. In the last figure we have considered some cases $(e=-5$, $f=2, \ldots, 3.2$ ) which are rather extreme, only to "activate" the other configurations. Therefore, for $f=2$ the favourable configurations are those with energy $-[(-e+2 f) / 4]$ and $-[(2-e+f) / 4]$. The zero-temperature magnetization per spin is between 0.25 and $0.5(m \approx 0.4)$. For $f=2.4,2.6,2.8$ we have only one configuration with energy $-[(-e+2 f) / 4]$, i.e., $m=0.5$ at $t=0$. In the case $f=3$ there are three favourable configurations with energy $-[(4+c+4 f) / 4],-[(-e+2 f) / 4]$ and $-[(2+3 f) / 4](m \approx 0.7)$. For $e=3.2$ the only configuration has the energy $-[(4+e+4 f) / 4]$, i.e., $m=1$ at $t=0$.

\subsection{The general case $\left(n_{1} \neq n_{2}\right)$}

To obtain numerically the variation of the magnetization versus the temperature, for different chain configurations and different values of $e$ and $f$, we have completed the iterative algorithm, used for the free energy, by the first derivatives (denoted with ') related to the reduced field $f$ of the following groups of quantities:

$$
\begin{aligned}
& c^{\prime}[0]=[0.5 k \operatorname{sh}(f / t)] / t, \\
& y^{\prime}[0]=[0.5 k \operatorname{ch}(f / t)] / t, \\
& x^{\prime}[0]=[0.5 k \operatorname{sh}(f / t)] / t, \\
& c^{\prime}[n]=2\left(y^{\prime}[0] y[n-1]+y[0] y^{\prime}[n-1]+c^{\prime}[0] c[n-1]+c[0] c^{\prime}[n-1]\right), \\
& y^{\prime}[n]=2\left(y^{\prime}[0] x[n-1]+y[0] x^{\prime}[n-1]+c^{\prime}[0] y[n-1]+c[0] y^{\prime}[n-1]\right), \\
& x^{\prime}[n]=2\left(x^{\prime}[0] x[n-1]+x[0] x^{\prime}[n-1]+y^{\prime}[0] y[n-1]+y[0] y^{\prime}[n-1]\right),
\end{aligned}
$$




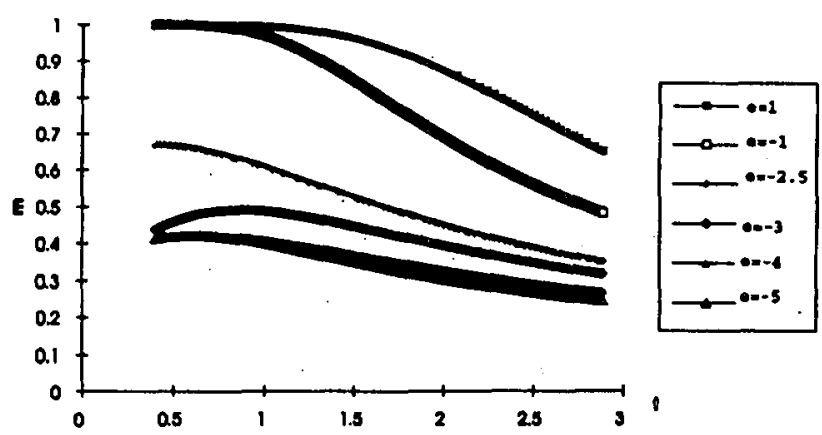

Fig. 6. Magnetization $m$ against the temperature for the chain configuration $n_{1}=2$, $n_{2}=1(f=1$ and $e=1,-1,-2.5,-3,-4,-5)$.

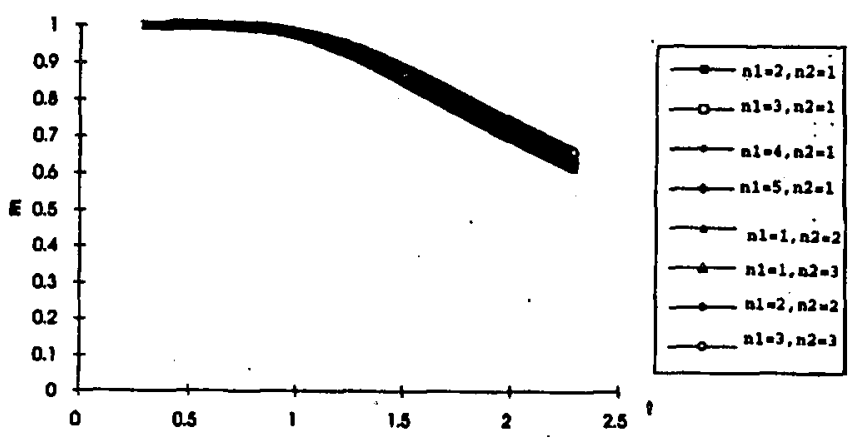

Fig. 7. The variation of $m$ versus $t$ for the particular value $e=-1$ is rather similar for different chain configurations (in this case $f=1$ ).

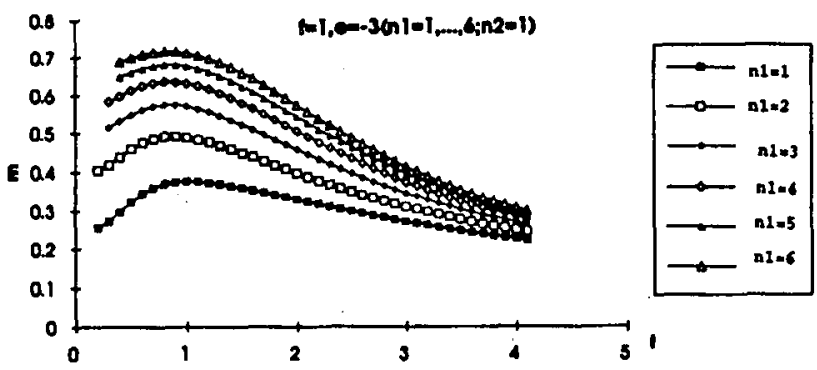

Fig. 8. Magnetization $m$ against $t$ for $f=1, e=-3$ and different chain configurations. The "blocks" of $n_{1}+n_{2}+2$ spins are composed of different tangled parts (with $\left.n_{1}=1,2,3,4,5,6\right)$ and the same one-spin untangled part $\left(n_{2}=1\right)$. 


$$
\begin{aligned}
& a_{q}^{\prime}=c^{\prime}[j]+2 y^{\prime}[j]+x^{\prime}[j], \\
& b_{q}^{\prime}=c^{\prime}[j]-x^{\prime}[j], \\
& d_{q}^{\prime}=c^{\prime}[j]-2 y^{\prime}[j]+x^{\prime}[j],
\end{aligned}
$$

with the symbol combinations: $\left(q=e, j=n_{1}\right)$ and $\left(q=0, j=n_{2}\right)$.

$$
\begin{aligned}
& A^{\prime}=\exp (e / t)\left(a_{0} a_{e}^{\prime}+a_{0}^{\prime} a_{e}\right)+\exp (-e / l)\left(b_{0} b_{e}^{\prime}+b_{0}^{\prime} b_{e}\right), \\
& B^{\prime}=\exp (e / t)\left(a_{e} b_{0}^{\prime}+a_{e}^{\prime} b_{0}\right)+\exp (-e / t)\left(b_{e} d_{0}^{\prime}+b_{e}^{\prime} d_{0}\right), \\
& C^{\prime}=\exp (-e / t)\left(b_{e} a_{0}^{\prime}+b_{e}^{\prime} a_{0}\right)+\operatorname{cxp}(e / l)\left(d_{e} b_{0}^{\prime}+d_{e}^{\prime} b_{0}\right), \\
& D^{\prime}=\exp (-e / t)\left(b_{e} b_{0}^{\prime}+b_{e}^{\prime} b_{0}\right)+\exp (e / l)\left(d_{e} d_{0}^{\prime}+d_{e}^{\prime} d_{0}\right) .
\end{aligned}
$$

Using an iterative algorithm based on the recurrence relations (3)-(6), (15)-(18) and the formulas (7), (12), (14) we can estimate numerically the values of the zero-temperature magnetization (with an accuracy limited by the lowest accessible temperature) and also, in general, we can study the variations of the magnetization $m$ against the temperature $t$ for different configurations (for different combinations of $n_{1}$ and $n_{2}$ ) and different values of $e$ and $f$. For their interpretation, we can analyse, as before, the ground-state configurations and the ground-state degeneracies, determining numerically the encrgy, the entropy and the magnetization at $t \approx 0$. In Fig. 6 there are represented some of these $(m-t)$ diagrams for the case: $n_{1}=2, n_{2}=1, f=1$ and different values of $e$, while in Fig. 7 there is illustrated the case $e=-1(f=1)$ for different configurations of the tangled chain. In the last figure the influence of chain configurations is relatively weak because for $e \geq-1$ when $t \rightarrow 0, m \rightarrow 1$, which is not the case, e.g., for $e=-3$ (Fig. 8).

\section{The susceptibility}

\subsection{The simple case $\left(n_{1}=n_{2}=1\right)$}

From the usual therrnodynamic relation the susceptibility is given by

$$
\chi \equiv \frac{\partial m}{\partial f}=m^{\prime}=\frac{\iota}{n_{1}+n_{2}+2}\left[\frac{\lambda_{\max }^{\prime \prime}}{\lambda_{\max }}-\left(\frac{\lambda_{\max }^{\prime}}{\lambda_{\max }}\right)^{2}\right],
$$

where $\lambda_{\max }^{\prime \prime}$ as a second derivative of $\lambda_{\max }$ related to $f$ is expressed through $A, B, C, D, A^{\prime}, B^{\prime}, C^{\prime}, D^{\prime}$ and also the second derivatives $A^{\prime \prime}, B^{\prime \prime}, C^{\prime \prime}, D^{\prime \prime}$ (with respect to $f$ and denoted with "). The very long expressions of these second derivatives will not be given here. Using these relations we can study the variation of $\chi$ versus the reduced temperature $t$ for different values of $e$ and $f$. Some of these $(\chi-t)$ diagrams are represented in Figs. 9 and 10. (The case $f=0$, represented in Fig. 10, at $t \rightarrow 0$ will be analysed more in detail a little after.) 


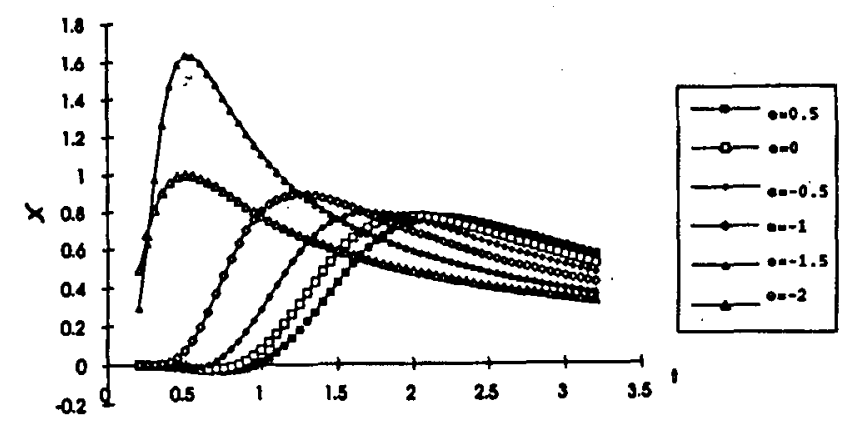

Fig. 9. Susceptibility $\chi$ against $t\left(n_{1}=n_{2}=1, f=0.5\right.$ and $\left.e=0.5,0,-0.5,-1,-1.5,-2\right)$.

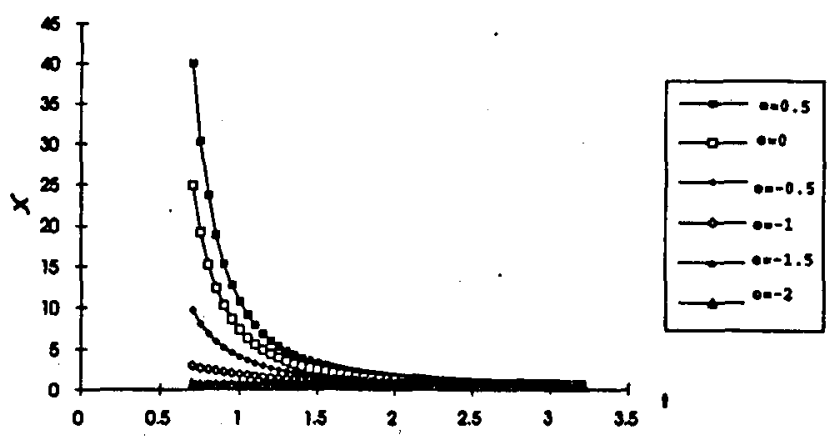

Fig. 10. Some $(\chi-t)$ diagrams in zero field $(f=0)$ for $n_{1}=n_{2}=1$ configuration and different value of $e(e=0.5,0,-0.5,-1,-1.5,-2)$.

In the case $n_{1}=n_{2}=1$ and $e=0$ from (19) and different expressions of $A, \ldots, D^{\prime \prime}$ we refind the simple relation of the unperturbed chain

$$
\chi=\left[k^{-4} \operatorname{ch}(f / t)\right] /\left[t\left(\operatorname{sh}^{2}(f / t)+k^{-4}\right)^{3 / 2}\right] .
$$

A very important feature is the zero-ficld susceptibility in the zero-temperature limit. From (11), (13) and the relations of the second derivatives, for $f=0$, we obtain

$$
\begin{aligned}
& A(0)=D(0)=4 \exp (-e / t)+\exp (e / t)\left(k^{2}+k^{-2}\right)^{2}, \\
& B(0)=C(0)=2[\exp (e / t)+\exp (-e / t)]\left(k^{2}+k^{-2}\right), \\
& A^{\prime}(0)=-D^{\prime}(0)=-4 \exp (e / t)\left(k^{4}+1\right) / t \\
& B^{\prime}(0)=-C^{\prime}(0)=4[\exp (-e / t)-\exp (e / t)] k^{2} / t, \\
& A^{\prime \prime}(0)=D^{\prime \prime}(0)=8\left[\exp (-e / t)+2 \exp (e / t) k^{4}+\exp (e / t)\right] / t^{2}, \\
& B^{\prime \prime}(0)=C^{\prime \prime}(0)=2[\exp (e / t)+\exp (-e / t)]\left(5 k^{2}+k^{-2}\right) / t^{2} .
\end{aligned}
$$


In the zero-temperature limit $(t \rightarrow 0)$ we have

$$
\begin{aligned}
& k=\exp (1 / t) \rightarrow \infty, k^{-1}=\exp (-1 / l) \rightarrow 0(J>0), \\
& k^{\prime}=\exp (e / t) \rightarrow \infty(e>0), k^{\prime}=1(e=0), k^{\prime}=\exp (e / t) \rightarrow 0(e<0) .
\end{aligned}
$$

Substituting the relations (20) in the explicit expression of (19), considering there the "highest power" term, based on the above conditions in the zerotemperature limit, we find ( (or $t \rightarrow 0$ ):

(a) $e=0, x \sim(1 / l) \exp (2 / t)$,

which is the susceptibility of an unperturbed chain of $N$ spins.

(b) $e>0, x \sim(2 / l) \exp (2 / t)$,

which is doubled, related to the susceptibility of an unperturbed chain.

(c) $e<0$

$\left(\mathrm{c}_{1}\right) e>-1, \chi \sim(2 / l) \exp [2(e+1) / l]$,

which is the double susceptibility of an unperturbed chain with a weaker nearest neighbours interaction.

$$
\left(c_{2}\right) e<-1, \chi \sim(13 / 6 t)
$$

or

$$
t \chi \sim \frac{13}{6}
$$

which, without this factor of $13 / 6$, is the susceptibility of the uncoupled paramagnetic spins (the simple Curie law).

$$
\left(c_{3}\right) e<-1, \chi \sim(1 / 4 t)
$$

or

$$
t \chi \sim \frac{1}{4}
$$

which is similar to the case $e=-1$, but the factor is very small ( 0.25 instead of $13 / 6)$.

Similar results, except the case $\left(\mathrm{c}_{3}\right)$, we find in [2], where the interpretation is done qualitatively using the concept of the local effective temperature. (If the local effective temperature is lowered, it keeps a certain group of spins always aligned together. The spins in each "frozen" block flip together as one large unit. Therefore, if any spin in the chain is correlated with one end of a frozen block, it is also correlated with the other end, and the spin correlation is enhanced. If the local effective temperature is raised, the chain is "melted" into disconnected pieces, decreasing drastically the spin correlation.) Therefore, in the case $\left(\mathrm{c}_{2}\right)$, following this interpretation, the raised effective temperatures have effectively "melted" the chain into uncoupled groups of spins. But, for the transition from $e=-1$ to $e<-1$ (very near to $e=-1$ ), associated with a change of the factor from $13 / 6$ to $1 / 4$, is rather difficult to give a local effective temperature interpretation.

In our paper we will try to give a comment of these results, relying on the ground-state configurations (at $f=0$ ) and the way of the evaluation of the 
correlation function [14, 15], without entering in details. (The complete study of the susceptibility through the correlation function is not the aim of this work.) In the case (b), with a state configuration $a$ (Fig. 5), the spins attempt to align themselves due their interactions in a direction which is determined by $J$ and $J^{\prime}$ (both ferromagnetic bonds), so two types of correlation terms will contribute to the susceptibility $\chi$, containing the multiple combinations of th $(1 / t)$ and $\operatorname{th}(e / l)$, with the similar behaviour at $t \rightarrow 0$. Therefore, $\chi$ becomes infinite when $t \rightarrow 0$, but with a coefficient which is twice as large as $\chi$ in the case $e=0$. (To understand the physical meaning for this situation we remember that in the unperturbed chain in $F$ the interaction energy is lowest when neighbouring spins are parallel, which tends to create correlations between all spins; however, statistical fluctuations raise the entropy, enough so that distant spins remain uncorrelated at all non-zero temperatures. In other words, for $1 \ll l \ll N, S_{i}$ and $S_{i+l}$ do not preferably take equal values, because the rather large number of configurations such that $S_{j} S_{j+1}=-1$ for $i<j<i+l$ prevents the order to set in between $i$ and $i+l$. Only at zero temperature does the chain become ordered, with $\left\langle S_{i} S_{i+l}\right\rangle$ finite as $l \rightarrow \infty$, and all spins then point in the same direction, either +1 or -1 . In the case of the tangled chain with $n_{1}=n_{2}=1$, the additional interaction $J^{\prime}(>0)$ between the first and the third spin in a box of four spins establish a supplementary condition of their ordering as one unit, increasing twicc the average correlation distance.)

When $e>-1$ (the case $\left(c_{1}\right)$ ) the situation is nearly the same, but in this case $\left(J^{\prime}<0\right)$ for every two spins $S_{i}$ and $S_{i+l}$ the number of configurations such that $S_{j} S_{j+1}=-1$ for $i<j<i+l$ is larger than before; the correlation distance is smaller. Also, considering the fact that again in this case the ground-state configuration is $a$ (Fig. 5) the three first spins in a box are acting as one unit with an interaction constant $J+J^{\prime}$ or $1+e$ in units of $J$. In the case $\left(c_{2}\right)$ with $e=-1$ there are three ground-state configurations with the same energy. The existence of certain clusters which can be inverted with no energy cost means that these clusters or groups of spins are uncoupled or independent; however, the possible presence of the configuration $a$ modifies the susceptibility of the independent spins by a factor $\xi$, which is just the average distance over which the spins can maintain the same direction [16]. In our simple case $\left(n_{1}=n_{2}=1\right)$ this factor is $\xi=13 / 6$. (This case is illustrated in Fig. 11, where there is represented the variation of $\chi \cdot t$ versus $t$, obtained numerically, with an accuracy limited by the lowest accessible temperature $t=0.08$. It is clearly visible from this figure that for $0.08 \leq t \leq 0.1$ the numerical fluctuations are present.) In the case $\left(c_{3}\right)$ with $e<-1$ the possible ground-state configurations are $b$ and $\boldsymbol{c}$ (Fig. 5) with the same energy. The groups of four spins (the microscopic spin clusters) are independent and their susceptibility in zero-field and $t \rightarrow 0$ is $\sim(1 / t)$, while the susceptibility per spin is $\chi \sim \frac{1}{4}(1 / l)$. (In the case $n_{1}=1, n_{2}=2$, where the ground-state configurations are similar, but with five spins in the microscopic clusters we have found numerically a constant $0.202 \approx \frac{1}{5}$, which is in entire accordance with our interpretation.)

It is clear from this comment that the discontinuous behaviour in $e=-1$ can be understood through the study of the ground-state configurations, as the consequence of flips of microscopic spin clusters. 


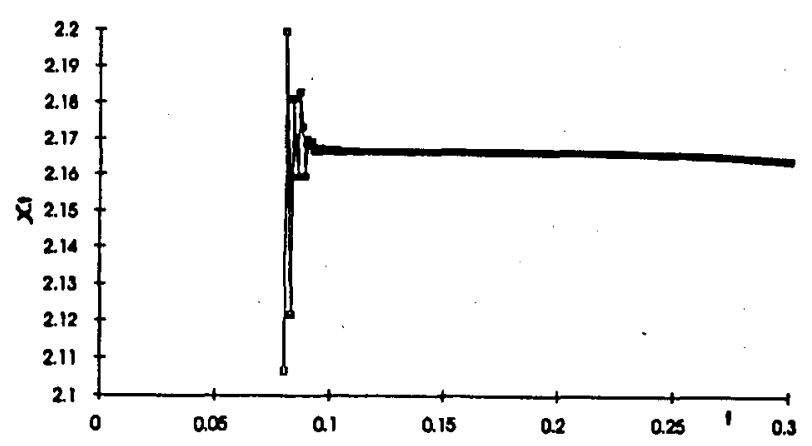

Fig. 11. $x \cdot t$ against $t$ for the particular case $e=-1\left(f=0\right.$ and $\left.n_{1}=n_{2}=1\right)$. The lowest temperature accessible numerically is $t=0.08$, but from this value to $t \approx 0.1$ small fluctuations are prescnt. However, it is quite clear that in this case $\xi \approx 2.167$.

\subsection{The general case $\left(n_{1} \neq n_{2}\right)$}

To obtain numerically the variation of the susceptibility versus the temperature, for different chain configurations and different values of $e$ and $f$, we have to complete the iterative algorithm used for the magnetization, by the second derivatives (denoted with ") with respect to the field $f$ of the following groups of quantities:

$$
\begin{aligned}
c^{\prime \prime}[0]= & \left(0.5 / t^{2}\right)[k \operatorname{ch}(f / l)], \\
y^{\prime \prime}[0]= & \left(0.5 / t^{2}\right)[k \operatorname{sh}(f / l)], \\
x^{\prime \prime}[0]= & \left(0.5 / t^{2}\right)[k \operatorname{ch}(f / t)], \\
c^{\prime \prime}[n]= & 2\left(y^{\prime \prime}[0] y[n-1]+y[0] y^{\prime \prime}[n-1]+2 y^{\prime}[0] y^{\prime}[n-1]\right. \\
& \left.\quad+c^{\prime \prime}[0] c[n-1]+c[0] c^{\prime \prime}[n-1]+2 c^{\prime}[0] c^{\prime}[n-1]\right), \\
y^{\prime \prime}[n]= & 2\left(y^{\prime \prime}[0] x[n-1]+y[0] x^{\prime \prime}[n-1]+2 y^{\prime}[0] x^{\prime}[n-1]\right. \\
& \left.\quad+c^{\prime \prime}[0] y[n-1]+c[0] y^{\prime \prime}[n-1]+2 c^{\prime}[0] y^{\prime}[n-1]\right), \\
x^{\prime \prime}[n]= & 2\left(x^{\prime \prime}[0] x[n-1]+x[0] x^{\prime \prime}[n-1]+2 x^{\prime}[0] x^{\prime}[n-1]\right. \\
& \left.\quad+y^{\prime \prime}[0] y[n-1]+y[0] y^{\prime \prime}[n-1]+2 y^{\prime}[0] y^{\prime}[n-1]\right), \\
a_{q}^{\prime \prime}= & c^{\prime \prime}[j]+2 y^{\prime \prime}[j]+x^{\prime \prime}[j], \\
b_{q}^{\prime \prime}= & c^{\prime \prime}[j]-x^{\prime \prime}[j], \\
d_{q}^{\prime \prime}= & c^{\prime \prime}[j]-2 y^{\prime \prime}[j]+x^{\prime \prime}[j],
\end{aligned}
$$

with the symbol combinations: $\left(q=e, j=n_{1}\right)$ and $\left(q=0, j=n_{2}\right)$.

$$
\begin{aligned}
& A^{\prime \prime}=\exp (e / t)\left(a_{0} a_{e}^{\prime \prime}+a_{0}^{\prime \prime} a_{e}+2 a_{0}^{\prime} a_{e}^{\prime}\right)+\exp (-e / l)\left(b_{0} b_{e}^{\prime \prime}+b_{0}^{\prime \prime} b_{e}+2 b_{0}^{\prime} b_{e}^{\prime}\right), \\
& B^{\prime \prime}=\exp (e / t)\left(a_{e} b_{0}^{\prime \prime}+a_{e}^{\prime \prime} b_{0}+2 a_{e}^{\prime} b_{0}^{\prime}\right)+\exp (-e / t)\left(b_{e} d_{0}^{\prime \prime}+b_{e}^{\prime \prime} d_{0}+2 b_{e}^{\prime} d_{0}^{\prime}\right), \\
& C^{\prime \prime}=\exp (-e / t)\left(b_{e} a_{0}^{\prime \prime}+b_{e}^{\prime \prime} a_{0}+2 b_{e}^{\prime} a_{0}^{\prime}\right)+\exp (e / t)\left(d_{e} b_{-}^{\prime \prime}+d_{e}^{\prime \prime} b_{0}+2 d_{e}^{\prime} b_{0}^{\prime}\right), \\
& D^{\prime \prime}=\exp (-e / t)\left(b_{e} b_{0}^{\prime \prime}+b_{e}^{\prime \prime} b_{0}+2 b_{e}^{\prime} b_{0}^{\prime}\right)+\exp (e / l)\left(d_{e} d_{0}^{\prime \prime}+d_{e}^{\prime \prime} d_{0}+2 d_{e}^{\prime} d_{0}^{\prime}\right) .
\end{aligned}
$$




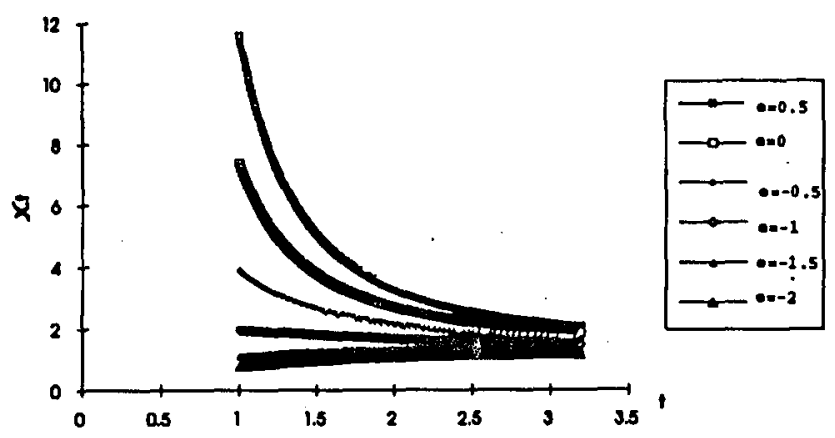

Fig. 12. Some $(x \cdot t-t)$ diagrams for the chain configuration $n_{1}=2, n_{2}=1(f=0$ and $e=0.5,0,-0.5,-1,-1.5,-2)$.
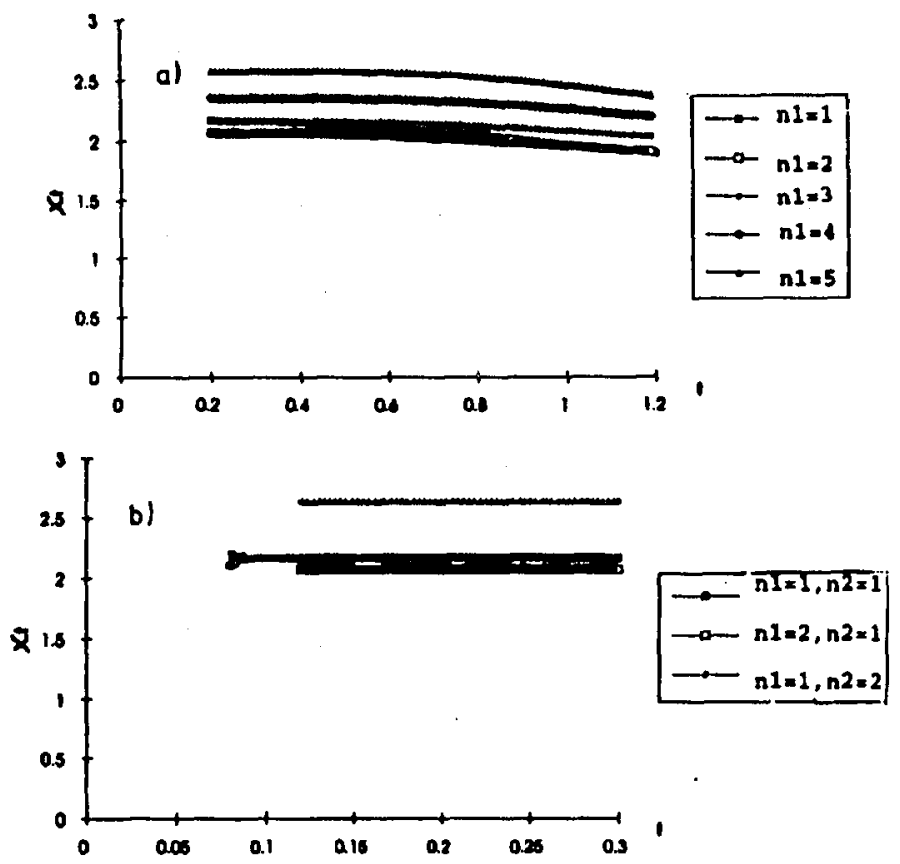

Fig. 13. $x \cdot t$ against $t$ for $e=-1$ and $f=0$. (a) Chain configurations are $n_{1}=1, \ldots, 5$ and $n_{2}=1$. (b) Chain configurations are $n_{1}=n_{2}=1, n_{1}=2, n_{2}=1$ and $n_{1}=1$, $n_{2}=2$.

Using an iterative algorithm based on the complete group of the recurrence relations and the explicit expression of $\chi$ (through $\lambda_{\max }, \lambda_{\max }^{\prime}$ and $\lambda_{\max }^{\prime \prime}$ ) we can study the variations of susceptibility $\chi$ against temperature $t$ for different 

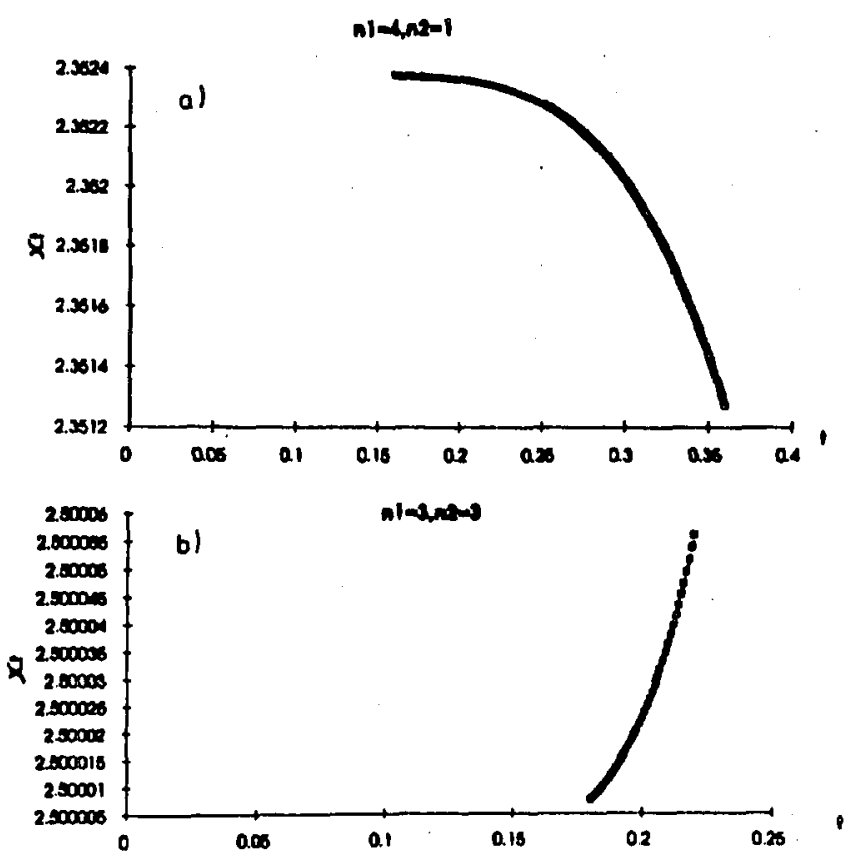

Fig. 14. The weak variation of $\chi^{\prime} t$ versus $t$ (when $t \rightarrow 0$ ) for $e=-1$ and $f=0$. (a) $\chi \cdot t$ is an increasing function when $t \rightarrow 0\left(n_{1}=4, n_{2}=1\right.$, i.e., $\left.n_{1}+n_{2}+2=7<8\right)$. (b) $x \cdot t$ is a decreasing function when $t \rightarrow 0\left(n_{1}=3, n_{2}=3\right.$, i.e., $\left.n_{1}+n_{2}+2=8\right)$.

combinations of $n_{1}, n_{2}, e$ and $f$. In Fig. 12, as illustration, there is represented one of these $(\chi \cdot t-t)$ diagram for the case $n_{1}=2, n_{2}=1, f=0$ and some values of $e$, while in Fig. 13a,b there are represented the variations of $\chi \cdot t$ versus $t$ for $f=0, e=-1$ and different combinations of $n_{1}$ and $n_{2}$. In Fig. $13 \mathrm{~b}$ we have taken a very narrow interval of $t$ to indicate that at $t \rightarrow 0$ the quantity $\chi \cdot t$ is a constant $\xi$. Therefore, it is possible to estimate numerically the value $\xi$ of the zero-field quantity $\chi \cdot t$ for $e=-1$ (at $t \rightarrow 0$ ) and different chain configurations, with an accuracy limited by the lowest accessible temperature. Some of these values are

$$
\begin{aligned}
& n_{1}=1, n_{2}=1, \approx 2.167, n_{1}=1, n_{2}=2, \approx 2.63, \\
& n_{1}=2, n_{2}=1, \approx 2.067, n_{1}=2, n_{2}=2, \approx 2.333, \\
& n_{1}=3, n_{2}=1, \approx 2.167, n_{1}=3, n_{2}=2, \approx 2.321, \\
& n_{1}=4, n_{2}=1, \approx 2.352, n_{1}=4, n_{2}=2, \approx 2.433, \\
& n_{1}=5, n_{2}=1, \approx 2.583, n_{1}=5, n_{2}=2, \approx 2.611 .
\end{aligned}
$$


We have also observed a different behaviour of $\chi \cdot t$ very close to zero temperature (in the case $e=-1, f=0$ ). From $n_{1}=1$ to $n_{1}=4$ and $n_{2}=1,2, \ldots$ we have found that for $n_{1}+n_{2}+2<8$ the correlation distance $\xi$ or the quantity $\chi \cdot t$ is an increasing function of $t$ (see e.g. Fig. 14a for $n_{1}=4, n_{2}=1$ ), which means that the ground-state configurations similar to $a$ become to be more probable at $t \rightarrow 0$. For $n_{1}+n_{2}+2>8$ the correlation distance is a decreasing function of $t$ (see e.g. Fig. 14b for $n_{1}=3, n_{2}=3$ ), which means that more probable (at $t \rightarrow 0$ ) are the other state configurations. From $n_{1} \geq 5$ the "magic" number 8 becomes 9 for the chain configurations with $n_{1}=5,10$ for the chain configuration with $n_{1}=6,11$ for $n_{1}=7$, etc.

\section{Conclusions}

In this paper, as in I, we have considered an Ising model on a tangled chain to understand the competition between ferromagnetic bonds of the usual chain and additional bonds of ferromagnetic or antiferromagnetic types, manifested in the behaviour of the magnetization and susceptibility. Using a modified matrix transfer method, some simple analytical calculations and an iterative algorithm, we have studied in detail this behaviour, for different configurations of composed parts (boxes) in the ferromagnetic tangled chain and different types of additional interactions. The proper explanation of the different $(m-t)$ diagrams is given in the "language" of ground state configurations and their degeneracies, which are sensitive to changes in the magnetic field (each metastable state is characterized by its energy and its magnetization per spin). Particularly very interesting is the behaviour of the susceptibility in zero-field $(f=0)$ and in zero-temperature limit $(t \rightarrow 0)$. A simple comment about this behaviour is given through the ground-state configurations and the correlation distance (correlation function). We have seen that the additional bonds, introduced as a form of perfectly disorder, can effect on the spin correlation breaking up the magnetic chain into independent segments. Concretely, the condition $e=-1$ or $J^{\prime}=-J$, between the ferromagnetic interaction $J$ along the chain and the antiferromagnetic interaction $J^{\prime}$ across the chain, plays a role of a "transition region" condition for this magnetic behaviour (from a coupled spin chain to another one composed by uncoupled groups of spins).

In the same way we can study also the case of an antiferromagnetic tangled chain with ferromagnetic or antiferromagnetic additional interactions. More interesting could be the competition between different bonds if we include more complicated pattern of chain configurations, e.g., if we consider a twisted chain.

\section{Acknowledgments}

The author is very grateful to Prof. R.B. Stinchcombe (Oxford) for his stimulating interest and very helpful orientation at the beginning of this work. He would like also to thank Prof. Abdus Salam, the International Atomic Energy Agency and UNESCO for the hospitality at the International Centre for Theoretical Physics, Trieste. 


\section{References}

[1] R. Mejdani, Acla:Phys. Pol. A 85, 907 (1994).

[2] J.C. Lee, J.M. Stephens, L.R. Mead, R.F. Joyce, Physica A 167, 749 (1990).

[3] R. Mejdani, preprint IC/93/67, ICTP, Trieste 1993.

[4] I. Harada, I. Mannari, M. Kaburagi, T. Tonegawa, J. Phys. Soc. Jpn. 62, 301 (1993).

[5] J.C. Lee, Physica A 160, 503 (1989).

[6] S. Masui, A.E. Jacobs, C. Wicentowich, B.W. Southern, J. Phys. A, Math. Gen. 26, 25 (1993).

[7] S. Masui, B.W. Southern, A.E. Jacobs, Pliys. Rev. B 39, 6925 (1989).

[8] B. Derrida, J. Vannimenus, Y. Pomeau, J. Phys. C, Solid Slate Phys. 11, 4749 (1978).

[9] U. Behn, V.B. Priezzhev, V.A. Zagrebnov, Physica A 167, 481 (1990).

[10] I. Morgenstern, K. Binder, R.M. Hornreich, Phys. Rev. B 23, 287 (1981).

[11] J. Villain, J. Phys. (France) 46, 1843 (1985).

[12] A. Moschel, K.D. Usadel, A. Hucht, Phys. Rev. B 47, 8676 (1993).

[13] M. Toda, R. Kubo, N. Saito, Slatislical Physics I, Springer-Verlag, Berlin 1992, Ch. 4.

[14] H.H. Chen, S. Ma, J. Slat. Phys. 20, 717 (1982).

[15] R. Balian, From Microphysics to Macrophysics I, Springer-Verlag, Berlin 1991, Ch. 9.

[16] S. Ma, Statistical Mechanics, World Scientific, Philadelphia 1985, Ch. 17. 